\title{
Pronóstico de la demanda turística de Chile basado en modelos lineales y no lineales estacionales
}

\author{
Cristian Colther* Ailin Arriagada-Millaman** \\ Universidad Austral de Chile (Chile)
}

\begin{abstract}
Resumen: En este trabajo se ha modelado la demanda turística que experimentó Chile en el período 2000- 2018 utilizando un modelo de regresión lineal con variables dicotómicas (MRL) y un modelo ARIMA con componente estacional (SARIMA). Los resultados muestran que el modelo SARIMA es más efectivo en replicar el comportamiento no-estacionario, no-lineal y con presencia de estacionalidad de las series, y es capaz de entregar pronósticos con un bajo error, en este caso de -5,6\% para el turismo emisivo y de -5,9\% para el turismo receptivo. Y, por tanto, puede ser una herramienta efectiva para pronosticar valores de la demanda turística en el corto plazo, y ayudar a la planificación y gestión del sector frente a fluctuaciones de la demanda.
\end{abstract}

Palabras Clave: Modelos ARIMA; Demanda turística; Estimación de corto plazo; Series no lineales; series estacionales.

\section{Forecast of Chile's tourism demand based on linear and non-linear seasonal models}

Abstract: TIn this paper, the Chilean tourism demand experienced during the period 2000-2018 has been modelled using a linear regression model with dichotomous variables (MRL) and an ARIMA model with a seasonal component (SARIMA). The results show that the SARIMA approach is more effective in replicating the non-stationary, non-linear behaviour and the presence of seasonality in the series, with the forecasts obtained from this model presenting a low rate of error, in this case $-5.6 \%$ for outbound tourism and $-5.9 \%$ for inbound tourism. In consequence, this approach may be an effective tool for forecasting forecast tourism demand in the short term, and support for planning and management in the sector in the face of fluctuations in tourism demand.

Keywords: ARIMA models; Tourism demand; Short-term estimation; Non-linear series; Seasonal series.

\section{Introducción}

La industria del turismo en estos últimos 10 años se ha convertido en un sector relevante en las economías a nivel internacional y en constante crecimiento en Latinoamérica, aportando al crecimiento económico y estimulando la movilidad de personas entre países. De acuerdo a la Organización Mundial del Turismo (OMT), el sector ha mostrado una gran expansión, pasando de los 674 millones de llegadas de turistas internacionales en el año 2000 a 1.322 millones en el año 2017, que representa un crecimiento de un 96\% en el período 2000-2017. El sector ha experimentado un crecimiento constante, a excepción de los shocks ocasionales provocados por crisis económicas, como la ocurrida en el año 2009 (OMT, 2017), ó de forma más reciente, la provocada por la pandemia vinculada al COVID-19 (Higgins-Desbiolles, 2020; Zenker \& Kock, 2020).

En el caso de Chile, la recepción de turistas ha crecido en forma importante, pasando de 1,8 millones en el año 2001 a 6,5 millones en el año 2017, representando un crecimiento del $267 \%$. Además, de acuerdo a cifras del Consejo Mundial de Viajes y Turismo, esta actividad generó de manera directa

\footnotetext{
* Universidad Austral de Chile (Chile); E-mail: cristian.colther@uach.cl; https://orcid.org/0000-0003-0606-6249

** Universidad Austral de Chile (Chile); E-mail: ailin.arriagada@gmail.com; https://orcid.org/0000-0003-3173-7438
} 
283 mil puestos de trabajos en el país, representando un 6,2\% de las exportaciones nacionales y una participación del 3,4\% en el PIB nacional del año 2017 (WTC, 2018).

Por los motivos mencionados anteriormente, el análisis del sector turismo ha cobrado relevancia; y en particular, el modelado de la demanda turística es una temática que ha captado el interés de los investigadores, para obtener pronósticos confiables respecto del comportamiento en el corto plazo que puede experimentar los flujos de turistas en un país (Song \& Li, 2008; Chatziantoniou et al., 2016; Goh \& Law, 2002, 2011; Lim \& McAleer, 2001; Song \& Li, 2008; Song et al., 2019; Song \& Witt, 2011; Qiu, $\&$ Park, 2019). Las estimaciones pueden ser muy útiles para tomar decisiones respecto de las medidas necesarias que puede tomar un país para responder a futuros aumentos o disminuciones en el flujo de turistas, en términos de capacidad de hospedaje, transportes públicos e infraestructura necesaria para su estancia y movilidad.

La presente investigación busca contribuir a un mejor entendimiento respecto del comportamiento de la demanda turística de Chile, utilizando un método lineal y uno no-lineal para pronosticar valores de demanda turística en el corto plazo, analizando el período 2001-2018. En el caso del método lineal se ha utilizado un modelo de regresión lineal con variables dicotómicas estacionales (MRL) y en el caso del modelo no-lineal se ha utilizado un modelo autorregresivo integrado de media móvil con componente estacional (SARIMA, acrónimo en inglés); de esta manera se espera obtener una metodología que permita realizar pronósticos a corto plazo de la demanda de turistas internacionales que llegan a visitar Chile (turismo receptivo), y de la demanda de turistas nacionales que visitan el extranjero (turismo emisivo). Este caso resulta de

interés, debido al importante crecimiento que ha experimentado el sector en los últimos años en Chile, y a la consolidación de sus destinos en la diversa oferta turística existente a nivel latinoamericano, fenómeno que requiere de herramientas que permitan darles una adecuada respuesta.

El artículo se desarrolla de la siguiente forma; comienza con una breve introducción de la temática y su importancia; la sección 2 presenta un resumen de las principales aportaciones de trabajos anteriores relacionados con pronósticos de demanda turística usando modelos SARIMA. La sección 3 describe la metodología y los datos utilizados, para luego presentar los principales resultados y su discusión en la sección 4. Finalmente se termina con la sección 5 de conclusiones, en donde se presentan los principales hallazgos del trabajo y recomendaciones en torno a políticas públicas aplicables al sector.

\section{Pronóstico de demanda turística con modelos SARIMA}

El pronóstico de la demanda turística es una temática que ha captado el interés de los investigadores, para obtener pronósticos confiables respecto del comportamiento en el corto plazo que puede experimentar los flujos de turistas en un país (Song \& Li, 2008; Song, Qiu, \& Park, 2019). Y la utilidad que puede tener este tipo de información para facilitar la planificación del sector, y anticiparse a los requerimientos futuros de los turistas en términos de infraestructura, servicios y transporte (Peiris, 2016).

Para modelar la demanda turística se han utilizado diferentes métodos, que varían desde aquellos que utilizan variables explicativas, como por ejemplo indicadores macroeconómicos u otra serie que se pueda considerar un factor de causa-efecto; hasta aquellos métodos, que analizan el comportamiento intrínseco de la serie y su comportamiento pasado para determinar lo que puede ocurrir en el futuro (Chatziantoniou et al., 2016; Goh \& Law, 2002, 2011; Lim \& McAleer, 2001; Song \& Li, 2008; Song et al., 2019; Song \& Witt, 2011).

Sin embargo, de la gran cantidad de métodos utilizados, diversos estudios han mostrado que los modelos SARIMA propuestos por Box y Jenkins (Box, et al., 2015; Box, Jenkins, \& MacGregor,

1974) presentan mejores resultados en pronosticar la demanda turística en diversos países, dada su capacidad de modelar series temporales, que habitualmente tiene un comportamiento no lineal, no estacionario en su crecimiento, e importantes fluctuaciones estacionales; en donde el comportamiento de los valores pasados determinan fuertemente el comportamiento de los valores futuros.

Estos modelos han sido ampliamente utilizados en otras áreas del conocimiento como la ingeniería y economía (Greene, 2018; Makridakis \& Hibon, 1997; Velásquez, Olaya, \& Franco, 2010), pero son de uso reciente en el área del turismo(Chang \& Liao, 2010; Choden \& Unhapipat, 2018; Du Preez \& Witt, 2003; Goh \& Law, 2002; Kim et al., 2011; Kulendran \& Witt, 2003; Kulendran \& Wong, 2005; Lim \& McAleer, 2000, 2002; Ma et al., 2016; Peiris, 2016; Song et al., 2019; Thushara, Su, \& Bandara, 2019; Witt, Witt, \& Wilson, 1994). 
En cuanto a la evolución del estudio de la demanda turística por medio de herramientas econométricas destaca el trabajo de Witt et al. (1994) quienes contrastan los resultados de 7 modelos para analizar la demanda turística del Reino Unido proveniente desde los principales países de origen de sus visitantes: Austria, Grecia, Italia y España. Sus resultados muestran que los modelos ARIMA permiten comprender mejor el comportamiento de la demanda turística y son los más efectivos para realizar pronósticos. Este resultado es obtenido para series con periodicidad mensual y trimestrales, y presenta baja tasa de error en el corto plazo.

Posterior al año 2000 se puede encontrar una mayor variedad de estudios, por ejemplo, Lim \& McAleer (2000) modelan la demanda de turistas asiáticos que visitan Australia, usando modelos SARIMA en series de periodicidad trimestral. En este caso, sus conclusiones muestran que los procesos SARIMA son apropiados para modelar la demanda turística debido a que ésta puede tener componentes estacionales deterministas o estocásticos, o ambos; y será, este procedimiento el más adecuado para representar datos que contengan un patrón estacional variable, en comparación al uso de un modelo lineal con variables ficticias estacionales.

Goh \& Law (2002) realizan un estudio en el cual contrastan 8 diferentes modelos de series de tiempo para pronosticar la demanda turística de Hong Kong considerando los 10 principales países de origen de sus visitantes. Analizan datos mensuales, evaluando la precisión de los pronósticos mediante diversos estadísticos de error. Sus resultados muestran que los pronósticos obtenidos utilizando modelos SARIMA tienen una menor tasa de error en sus pronósticos que los demás métodos considerados, debido a que capturan tanto la estacionalidad no estacionaria como el comportamiento estocástico de las series. Lim \& McAleer (2002), por su parte, logran establecer los mejores modelos ARIMA y SARIMA para pronosticar la demanda de turistas internacionales provenientes de Hong Kong, Malasia y Singapur que visitan Australia. Utilizando series temporales con datos trimestrales concluyen que los modelos SARIMA permiten obtener mejores pronósticos para los casos de Hong Kong y Malasia.

Los resultados indican que el modelo SARIMA suele ser el mejor modelo para realizar pronósticos de la demanda turìstica futura, además de contar con el beneficio de ser relativamente menos complejo en comparación con otros modelos de análisis de series de tiempo. Du Preez \& Witt (2003), por ejemplo, comparan modelos de series temporales estacionarias en espacio de estados univariantes y multivariantes con el modelo SARIMA univariante para pronosticar la demanda turística de las Seychelles proveniente desde Francia,

Italia, Alemania y Reino Unido, utlizando datos mensuales. Sus resultados muestran que los modelos SARIMA pueden superar a los modelos multivariados, entregando mejores pronósticos, y con una tasa de error menor. Kulendran \& Witt (2003) estudian siete modelos de pronóstico, incluidos los modelos de media móvil integrada autorregresiva (ARIMA) aplicado a la demanda de turismo de negocios internacionales de Japón, Nueva Zelanda, el Reino Unido y los Estados Unidos que visitaron Australia, analizando datos de frecuencia trimestral, evaluando el grado de precisión de los pronósticos en un horizonte de 1, 4 y 6 trimestres. Sus resultados muestran que el rendimiento de pronóstico relativo de los modelos depende en gran medida de la longitud del horizonte de pronóstico y que los modelos SARIMA muestran un bajo error en horizontes de corto plazo (1 y 4 trimestres).

Profundizando el estudio, se ha logrado identificar algunas características bajo las cuales aumenta el grado de precisión de las predicciones obtenidas a partir de modelos SARIMA, como la correcta identificación del comportamiento estacional de la serie, la aplicación de primeras diferencias y el horizonte de pronóstico que debe ser de corto plazo. Kulendran \& Wong (2005) modelan la demanda turística australiana de visitantes provenientes del Reino Unido, usando diversas formas estacionales para los modelos ARIMA, analizando datos trimestrales diferenciando a turistas de vacaciones y de negocios. Sus resultados muestran que la selección del modelo más preciso debe basarse en el rendimiento del pronóstico fuera de la muestra, y que el rendimiento de este pronóstico depende de la naturaleza de la variación estacional en las series temporales; donde la identificación correcta de la variación estacional puede mejorar el rendimiento de pronóstico del modelo ARIMA, siendo los modelos SARIMA, los que permiten obtener mejores predicciones para el comportamiento de los turistas de vacaciones en el corto plazo.

Chang \& Liao (2010) usan los modelos SARIMA para pronosticar las salidas mensuales de turismo emisor en Taiwán y utilizan dos estadísticos de error (MAPE y MAE) para medir la precisión del pronóstico. Sus resultados muestran que se debe trabajar con la primera diferencia no estacional para obtener una tendencia determinista en las series de turismo emisor, acción necesaria para obtener modelos SARIMA válidos. Los modelos estimados son adecuados para pronosticar el patrón estacional en los datos con un bajo error, menor al 8\%. Kim et al., 2011 evalúan el desempeño de los intervalos de 
predicción generados a partir de 5 diferentes modelos aplicados a la demanda de turistas que llegan a Hong Kong y Australia en un horizonte de pronóstico de 1 a 12 períodos. Sus resultados muestran que los modelos SARIMA son efectivos para pronósticos en el corto plazo, menor a 4 períodos, con intervalos de confianza acotados, y tiende a diverger subestimando los valores pronosticados en la medida que se aumenta el horizonte de pronóstico.

El uso de modelos SARIMA para la estimación se ha preferido para estudiar el comportamiento de la demanda turística. Ma et al. (2016) profundizan en el estudio de la demanda de turistas chinos para Australia utilizando modelos ARIMA con componente estacional. Analizan series con periodicidad mensual para determinar pronósticos de demanda a un horizonte de 12 períodos. Sus resultados muestran que los modelos SARIMA muestran un buen desempeño en cuanto a pronósticos en el corto plazo, con tasa de error en general menor al 10\%, capaz de pronosticar en forma adecuada la estacionalidad inherente a las series temporales de demanda turística. Asimismo, Peiris (2016) utiliza un modelo SARIMA para pronosticar la llegada de turistas internacionales a Sri Lanka, con series de periodicidad mensual, y determina la precisión del pronóstico con diferentes estadísticos de error. Sus resultados muestran que el modelo SARIMA es adecuado para pronosticar la llegada de turistas a Sri Lanka con un error inferior al $10 \%$.

Choden \& Unhapipat (2018) modelan la demanda turística de Bután analizando datos mensuales, de los visitantes internacionales. Sus resultados muestran que el modelo SARIMA puede pronosticar con un 91\% de precisión, en un horizonte de pronostico de 6 meses. Thushara, Su, \& Bandara (2019) modelan la demanda turística que experimenta Sri Lanka utilizando modelos SARIMA, analizando datos mensuales de sus 10 principales países de origen de los turistas visitantes y realizando pronósticos a un horizonte de 12 meses. En este caso, el método mostro ser muy útil para predecir la demanda turística en presencia de estacionalidad en la demanda presentando una tasa de error menor al 10\%.

En 2019, Song et al. evalúan nuevamente la precisión de seis metodologías para la estimación de la demanda turística de Hong Kong, utilizando reglas de puntuación para evaluar la densidad de los pronósticos generados por estos métodos. La densidad de pronósticos permite conocer la probabilidad de ocurrencia de un cierto resultado en particular. Utilizando datos trimestrales para el análisis, sus resultados respaldan el buen desempeño de los modelos SARIMA, especialmente para pronósticos de corto plazo en un horizonte de 6 a 8 trimestres.

\section{Metodología}

Para proceder con el estudio, se utilizaron datos obtenidos de la Subsecretaria de Turismo (Subsecretaria de Turismo de Chile, 2018) de los flujos turísticos para el período 2000-2018 con información trimestral, por lo que la muestra consta de 76 observaciones trimestrales (ver anexo), y realizar pronósticos a corto plazo de la demanda de turistas internacionales que llegan a visitar Chile (turismo receptivo), y de la demanda de turistas nacionales que visitan el extranjero (turismo emisivo).

Para el análisis se ha utilizado un método lineal y uno no lineal para modelar las series y obtener pronósticos en el corto plazo. En el caso del método lineal se ha utilizado un modelo de regresión lineal con variables dicotómicas estacionales (MRL), que permiten modelar una serie temporal, con una componente estacional presente, de la siguiente forma (ver ecuación 1),

$$
y_{t}=\beta_{0} \cdot t+\beta_{1} D_{1}+\beta_{2} D_{2}+\beta_{3} D_{3}+\beta_{4} D_{4}+e_{t}
$$

Donde $D 1, D 2, D 3, D 4$ son variables dicotómicas asociadas a cada trimestre del año y et es la componente aleatoria normal.

En el caso del modelo no-lineal, se ha utilizado un modelo autorregresivo integrado de media móvil con componente estacional (SARIMA, acrónimo en inglés), método que relaciona los valores presentes de una serie con sus valores pasados, en ecuaciones dinámicas autoregresivas no lineales y componentes estocásticos correlacionados; pero con varianza y media constantes, a pesar de que puede tener componentes estacionales. El modelo se define

en la ecuación (2) (Box et al., 2015): 


$$
\left(1-\phi_{1} B-\ldots-\phi_{p} B^{p}\right)(1-B)^{d} y_{t}=\left(1-\theta_{1} B-\ldots-\theta_{q} B^{q}\right) e_{t}
$$

y es habitual resumirlo de la siguiente forma $\operatorname{ARIMA}(p, d, q)$ donde $\mathrm{p}$ es el orden del proceso autoregresivo considerado, $d$ es el número de diferencias que se han aplicado a la serie para que ésta sea de comportamiento estacionario, y $q$ es el orden del proceso de media móvil considerado.

Las series temporales también puede presentar una componente estacional, que se observa cuando ocurren patrones de comportamiento regulares que se repiten cada $S$ períodos, siendo $S$ es el número de períodos que deben pasar hasta que vuelva a iterar el patrón, definiendo un modelo ampliado denominado SARIMA, y se expresa de la forma $\operatorname{ARIMA}(p, d, q) \mathrm{x}(P, D, Q)$, donde $P$ se asocia al elemento autoregresivo estacional, $D$ a la diferenciación estacional y $Q$ al

componente MA estacional según la ecuación (3),

$$
\phi_{p}(B) \Phi_{P}(1-B)^{d}\left(1-B^{s}\right)^{D} y_{t}=\theta_{q}(B) \Theta_{Q}\left(B^{s}\right) e_{t}
$$

Donde $\phi_{p}(B)=\left(1-\phi_{1} B-\ldots-\phi_{p} B^{p}\right)$ orden del proceso autoregresivo no estacional;

$$
\begin{aligned}
& \Phi_{P}\left(B^{s}\right)=\left(1-\Phi_{1} B-\ldots-\Phi_{P} B^{s}\right) \text { orden del proceso autoregresivo estacional; } \\
& \theta_{q}(B)=\left(1-\theta_{1} B-\ldots-\theta_{q} B^{q}\right) \text { orden del proceso de media móvil no estacional; } \\
& \Theta_{Q}\left(B^{s}\right)=\left(1-\Theta_{1} B-\ldots-\Theta_{Q} B^{s}\right) \text { orden del proceso de media móvil estacional; }
\end{aligned}
$$

$(1-B)^{d}$ diferencia no estacional de orden $d$;

$\left(1-B^{S}\right)^{D}$ diferencia estacional de orden $D$;

+ término de error $\sim \operatorname{iid}\left(0, \sigma^{2}\right) ; B$ operador de diferenciación; $S$ orden de estacionalidad.

En este caso, el modelo recoge las componentes que se vinculan a la existencia de una componente estacional en su parte autoregresiva o en su parte de media móvil. Los modelos ARIMA proporcionan un marco útil para comprender cómo se generan las series temporales de turismo, sin embargo, el enfoque SARIMA requiere que se pruebe la no estacionariedad de la serie temporal antes de realizar el ejercicio de estimación y pronóstico.

Si una serie no es estacionaria (es decir, la serie tiene una media y una varianza que no son constantes en el tiempo), la serie tiene que diferenciarse para transformarla en una serie estacionaria para su correcto modelamiento, y con esto generar pronósticos válidos. Los modelos SARIMA pueden ser usados para estimaciones de corto plazo en series temporales sin restricciones de la periodicidad de sus datos (trimestral, semestral, mensual, etc.); que, en este caso, $S$ toma el valor 4 , dado que las series analizadas son de periodicidad trimestral.

Para evaluar el grado de precisión de los modelos estimados, se han reservado los últimos 8 trimestres (2017-2018) para comparar los valores obtenidos de los modelos, con los datos reales sucedidos en esos trimestres, y así medir el error cometido utilizando algunos estadísticos que son de uso habitual como son: error medio (EM), raíz del error cuadrático medio (RECM), error absoluto medio (EAM), porcentaje de error medio (PEM), porcentaje de error absoluto medio (PEAM), U de Theil (Goh \& Law, 2002).

\section{Resultados y discusión}

En la figura 1 se puede observar el comportamiento de la demanda del turismo emisivo y receptivo de Chile para el período 2000-2016. En este caso, ambas series temporales presentan un comportamiento creciente así como un componente estacional que varían en el tiempo. 
Figura 1: Número de turistas que ingresaron y que salieron de Chile (2000-2016).

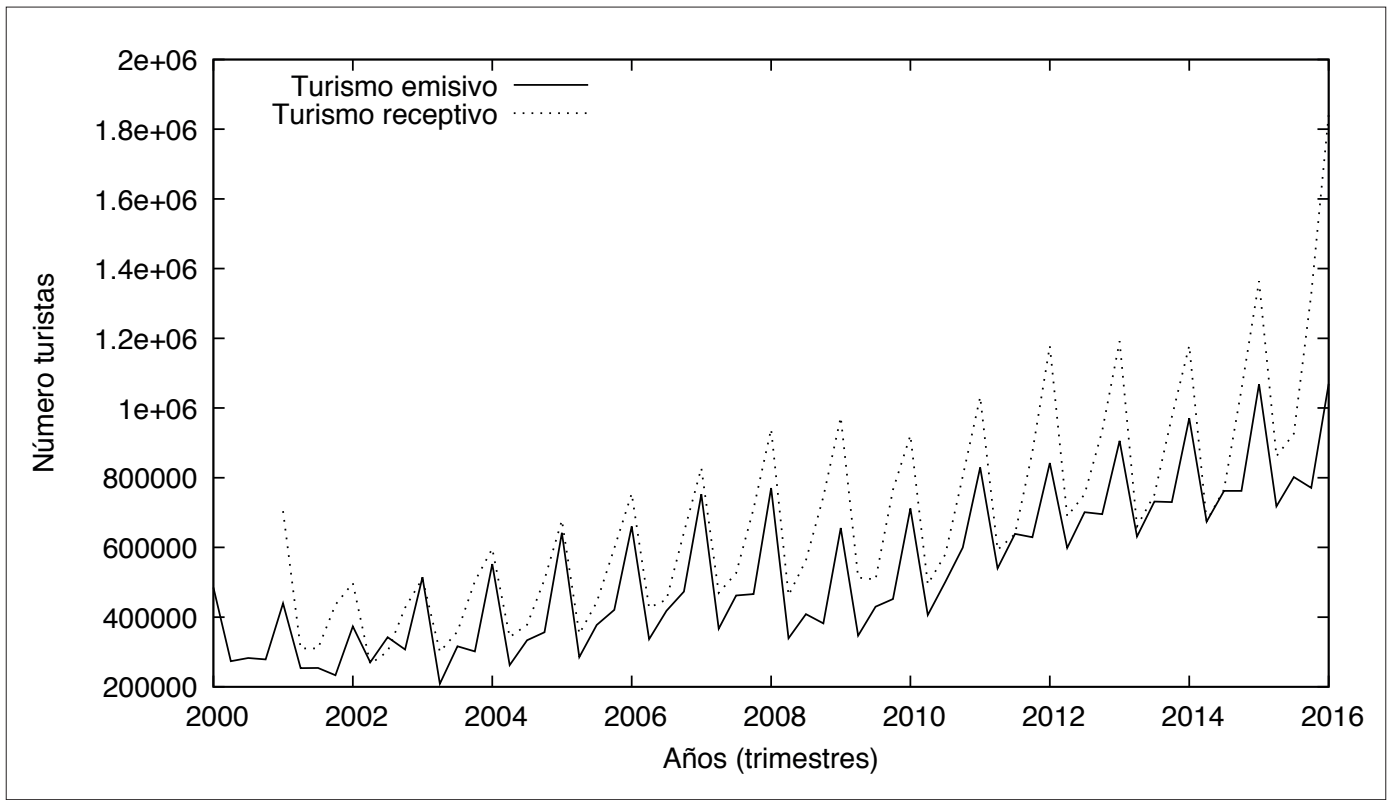

Fuente: Elaboración propia de los autores en base a datos de Subsecretaria de Turismo de Chile.

Para realizar un análisis del comportamiento de crecimiento de las series se puede observar la figura 2 , en donde se encuentran representadas las tasas de crecimiento interanual para el turismo emisivo y el receptivo.

Figura 2: Tasa interanual de turistas que ingresaron y que salieron de Chile (2000-2016).

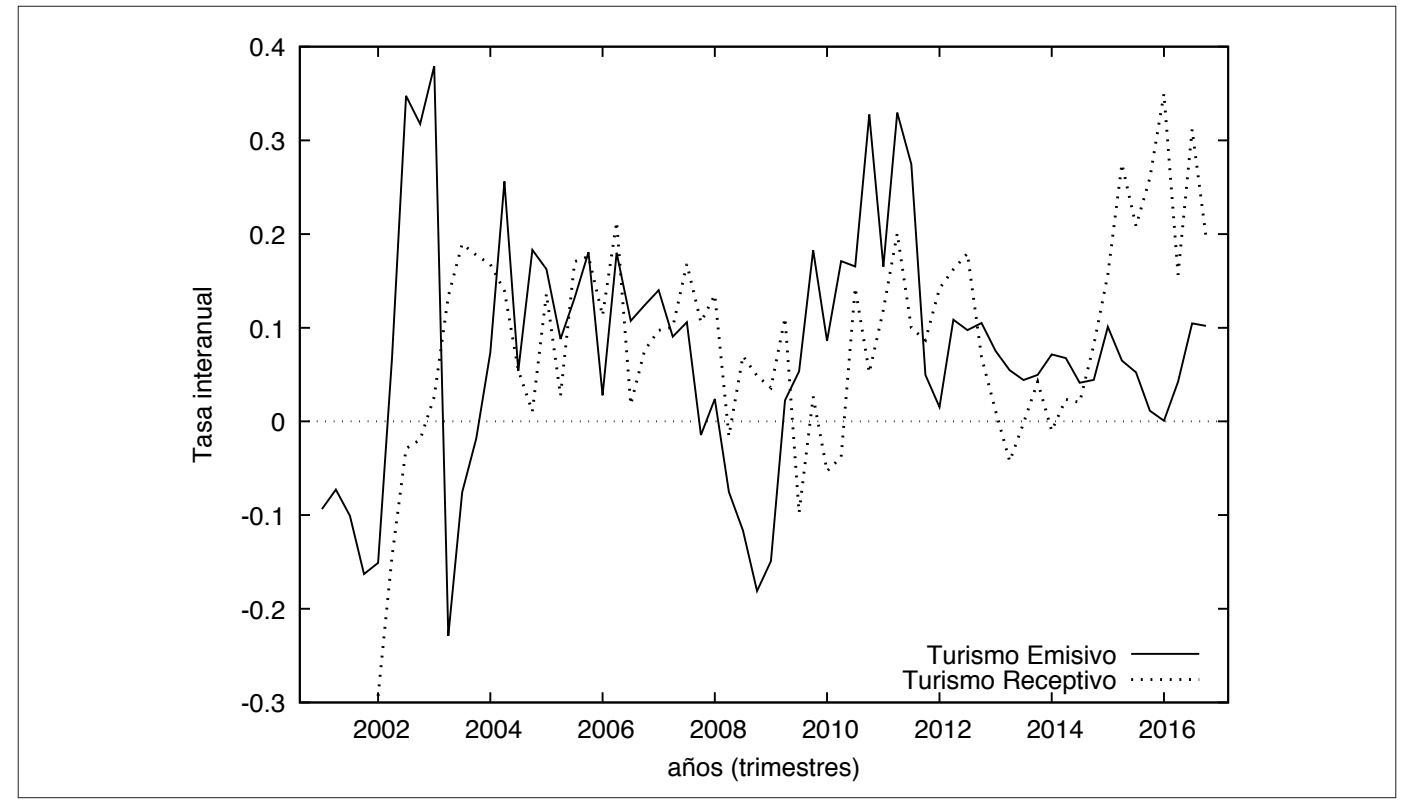

Fuente: Elaboración propia de los autores en base a datos de Subsecretaria de Turismo de Chile. 
En este caso, se puede ver que, entre el año 2004 y 2008, el turismo emisivo y receptivo experimentaron un crecimiento constante con una tasa promedio de crecimiento de $12 \%$, comportamiento que se desacopla en el año 2009, posterior a la crisis financiera de nivel internacional que afectó también a Chile, impactando en forma negativa al turismo emisivo el cual presenta tasas negativas de crecimiento, y en forma positiva al turismo receptivo. También se puede observar un decrecimiento importante del turismo receptivo en 2010, que pudo deberse a los efectos del terremoto ocurrido en febrero de ese año, y que afectó a un grupo importante de las regiones del sur de Chile.

Desde el año 2010 en adelante, se puede ver que las series de tasas de crecimiento interanual se desfasan mostrando un leve retraso el turismo receptivo respecto del emisivo. Además, entre los años 2010 y 2015, se puede observar un crecimiento constante de ambas series, pero con una tasa promedio mayor entre los años 2010 y 2012 , de un $17 \%$ aproximadamente, para luego mostrar una tasa de crecimiento menor, de un 7\% aproximadamente. En el año 2016, se puede observar un comportamiento opuesto entre las tasas de crecimiento de las series analizadas, en donde, cuando el turismo receptivo crece, el turismo emisivo decrece, situación que coincide con un período de bajo crecimiento del PIB chileno y el empeoramiento en los términos de intercambio relativos. Del análisis gráfico, se puede ver claramente el comportamiento no estacionario de las series en su crecimiento, así como su estacionalidad.

Como primera aproximación se han modelado las series de turismo emisivo y receptivo por medio de una regresión lineal en el tiempo con variables dicotómicas para modelar la componente estacional. Los coeficientes estimados se pueden ver en la tabla 1 para el turismo emisivo y receptivo respectivamente.

\section{Tabla 1: Modelo de regresión lineal con componente estacional (MRL) del turismo emisivo y receptivo.}

\begin{tabular}{|c|c|c|c|c|c|c|c|c|}
\hline & \multicolumn{4}{|c|}{ Turismo emisivo } & \multicolumn{4}{c|}{ Turismo receptivo } \\
\hline Variables & Estimación & $\begin{array}{c}\text { Std. } \\
\text { error }\end{array}$ & $\begin{array}{c}\mathbf{t} \\
\text { value }\end{array}$ & p-value & Estimación & $\begin{array}{c}\text { Std. } \\
\text { error }\end{array}$ & $\begin{array}{c}\mathbf{t} \\
\text { value }\end{array}$ & p-value \\
\hline tiempo & 9380 & 363.4 & 25.80 & $<0.0001$ & 13821 & 781.8 & 17.68 & $<0.0001$ \\
\hline D1 & 410538 & 18631.5 & 22.03 & $<0.0001$ & 465516 & 39747.2 & 11.71 & $<0.0001$ \\
\hline D2 & 108015 & 18867.8 & 5.73 & $<0.0001$ & 28221 & 40289.3 & 0.70 & 0.4864 \\
\hline D3 & 180294 & 19108.0 & 9.44 & $<0.0001$ & 80307 & 40839.2 & 1.96 & 0.054 \\
\hline D4 & 174452 & 19352.2 & 9.02 & $<0.0001$ & 280360 & 41396.6 & 6.77 & $<0.0001$ \\
\hline n & 68 & & & & 64 & & & \\
\hline R-squared & 0.93 & & & & 0.88 & & & \\
\hline Adjunted R- & 0.93 & & & & 0.93 & & & \\
\hline squared & & & & & & & & \\
\hline F(4,63) & 224.2 & & & $1.68 \mathrm{e}-36$ & 112.5 & & & \\
\hline
\end{tabular}

Fuente: Elaboración propia de los autores en base a datos de Subsecretaria de Turismo de Chile.

De la tabla 1 se puede apreciar que, en el caso del turismo emisivo, todos los coeficientes resultan significativos; y por tanto, la estacionalidad es un fenómeno que afecta en todos los trimestres del período, pero con diferentes intensidades. Además, se puede ver que la componente estacional más relevante sucede en el primer trimestre de cada año, trimestre que coincide con el aumento del flujo de turistas por causa de los feriados laborales por vacaciones.

La segunda componente estacional relevante ocurre en el tercer trimestre, que coincide en Chile con los feriados laborales producto de la celebración de la independencia del país y de los festivos por vacaciones escolares de invierno.

En el caso del Turismo receptivo, se puede ver en la tabla 1 que ahora sólo dos trimestres presentan coeficientes significativos, y por tanto existe un comportamiento estacional; en este caso, el primer y 
el cuarto trimestre la demanda experimenta aumentos significativos en términos del flujo de turistas, además del aumento constante de turistas en cada año. Estos trimestres coinciden con las vacaciones de invierno en el hemisferio norte y con las vacaciones de los países latinoamericanos vecinos (particularmente Argentina).

En todo caso, se debe mencionar que aún cuando se pueden utilizar los modelos de regresión lineal para modelar las series temporales y realizar pronósticos, y a pesar del alto grado de explicación que presentan los modelos MRL (R2 ajustado =0,93); estos modelos no son válidos, dado que el residuo obtenido en ambos casos, no cumple con tener un comportamiento aleatorio normal, y presenta también una componente de autocorrelación en el residuo, que no puede ser capturado por los modelos lineales. Es por este motivo, que una alternativa más adecuada para modelar la demanda turística son los modelos SARIMA.

En la tabla 2 se puede ver los resultados de la estimación de modelos ARIMA con componente estacional aplicado al turismo emisivo y receptivo.

Tabla 2: Modelo SARIMA Turismo emisivo y receptivo.

\begin{tabular}{|c|c|c|c|c|c|c|c|c|}
\hline & \multicolumn{4}{|c|}{ Turismo emisivo } & \multicolumn{4}{c|}{ Turismo receptivo } \\
\hline Variables & Estimación & D.E. & estd. z & p-valor & Estimación & S.D. & estd. z & p-valor \\
\hline \multicolumn{1}{|c|}{$\phi$} & -0.34 & 0.15 & -2.27 & 0.0235 & -0.45 & 0.11 & -4.13 & $<0.0001$ \\
\hline \multicolumn{1}{|c|}{} & -0.32 & 0.15 & -2.15 & 0.0315 & & & & \\
\hline $\mathrm{n}$ & 63 & & & 59 & & & & \\
\hline $\begin{array}{l}\text { Criterio } \\
\text { de } \\
\text { Schwartz }\end{array}$ & 1540.5 & & 1490.5 & & & & \\
\hline $\begin{array}{l}\text { Criterio } \\
\text { de } \\
\text { Akaike }\end{array}$ & 1534.1 & & 1486.4 & & & & \\
\hline
\end{tabular}

Fuente: Elaboración propia de los autores en base a datos de Subsecretaria de Turismo de Chile.

Los modelos estimados necesitaron de procesos de diferenciación normal y estacional, para obtener series de comportamiento estacionario, el cual es un supuesto básico para obtener modelos ARIMA con componente estacional válidos. Para analizar si las series son estacionarias en media y en varianza, se ha aplicado el test aumentado de Dickey-Fuller (ADF) para las series en logaritmo y para su primera diferencia, determinándose que para ambas series, sus primeras diferencias son estacionarias en media y varianza. En ambos casos, se han estimado modelos que presentan una componente de paseo aleatorio, es decir, un comportamiento de crecimiento constante, además de una componente autorregresiva estacional de primer orden que expresa el comportamiento estacional de las series en el tiempo.

En el caso del turismo emisivo se ha estimado un modelo $\operatorname{SARIMA}(0,1,1)(0,1,1)$ que presenta una componente de media móvil aleatorio normal y estacional del residuo. En el caso del turismo receptivo, se ha estimado un modelo $\operatorname{SARIMA}(0,1,1)(0,1,0)$, que sólo presenta una media aleatoria normal en su residuo, este es más complejo que el anterior, debido a que la serie presenta una volatilidad mayor en su crecimiento de tendencia de largo plazo, y una componente estacional que fluctúa en el tiempo.

Para ambos modelos se han realizado los contrastes estadísticos que aseguran la significancia de los parámetros estimados y la confiabilidad del modelo. Además, el comportamiento de los residuos de ambos modelos es aleatorio normal sin componentes autorregresivas y componentes periódicas presentes en ellos; es decir, que no hay evidencias contra la hipótesis de ausencia de autocorrelación de los residuos, como tampoco contra la hipótesis de normalidad de los residuos al nivel de significancia del $1 \%$. Los resultados de las predicciones se pueden observar en la figura 3. 
Figura 3: Pronóstico del Turismo Emisivo y Receptivo según los modelos MRL y SARIMA para el periodo 2017-2018.

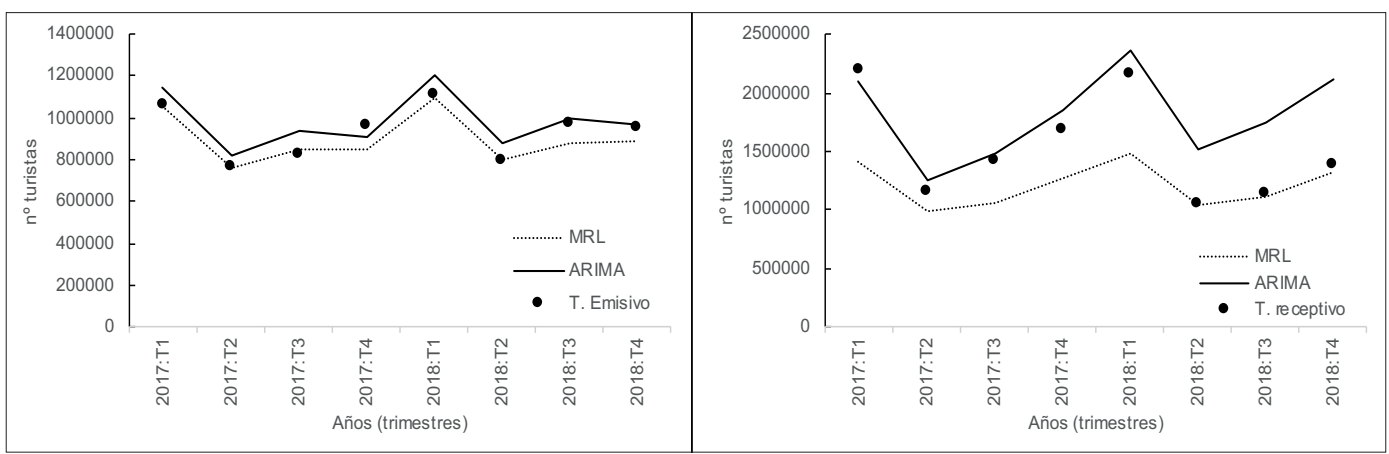

Fuente: Elaboración propia de los autores en base a datos de Subsecretaria de Turismo de Chile.

De la figura 3 se puede ver que, dadas las características particulares de cada serie, los modelos son más eficaces en el caso del turismo emisivo que en el receptivo; esto se puede deber a la mayor volatilidad y crecimiento no lineal que experimenta el turismo receptivo. En el caso del turismo emisivo los modelos MRL y SARIMA generan pronósticos muy similares, cometiendo errores que se pueden considerar por defecto y exceso, los cuales no se encuentran muy alejados de los valores reales. En cambio, en el caso del turismo receptivo, los modelos presentan resultados diferentes; el modelo MRL tiene errores significativos en los primeros 5 trimestres y luego converge en sus pronósticos a los valores reales, en cambio el modelo SARIMA pronostica valores muy cercanos a los valores reales en los primeros 5 trimestres y luego divergen sus resultados respecto de los valores reales. En las tablas 3 y 4 se puede ver las estimaciones de los errores cometidos en cada valor pronosticado respecto de los datos reales para cada trimestre.

Tabla 3: Resultados de pronósticos entregados por modelos para turismo emisivo (8 trimestres futuros).

\begin{tabular}{|l|l|l|l|l|l|}
\hline \multicolumn{1}{|c|}{ Tiempo } & \multicolumn{1}{|c|}{$\mathbf{N}^{\mathbf{o}}$ turistas } & \multicolumn{1}{|c|}{ MRL } & \% error MRL & \multicolumn{1}{|c|}{ SARIMA } & \multicolumn{1}{c|}{$\begin{array}{c}\text { error } \\
\text { SARIMA }\end{array}$} \\
\hline 2017:T1 & 1065600 & 1057800 & 0.7 & 1141620 & -7.1 \\
\hline 2017:T2 & 763502 & 764661 & -0.2 & 817542 & -7.1 \\
\hline 2017:T3 & 828179 & 846321 & -2.2 & 938912 & -13.4 \\
\hline 2017:T4 & 962770 & 849860 & 11.7 & 907595 & 5.7 \\
\hline 2018:T1 & 1108400 & 1095330 & 1.2 & 1199870 & -8.3 \\
\hline 2018:T2 & 794784 & 802184 & -0.9 & 875791 & -10.2 \\
\hline 2018:T3 & 968758 & 883844 & 8.8 & 997160 & -2.9 \\
\hline 2018:T4 & 953124 & 887383 & 6.9 & 965843 & -1.3 \\
\hline
\end{tabular}

Fuente: Elaboración propia de los autores en base a datos de Subsecretaria de Turismo de Chile. 


\section{Tabla 4: Resultados de pronósticos entregados por modelos MRL y SARIMA para el turismo receptivo ( 8 trimestres futuros).}

\begin{tabular}{|l|r|r|r|r|r|}
\hline \multicolumn{1}{|c|}{ Tiempo } & \multicolumn{1}{c|}{$\mathbf{N}^{\mathbf{o}}$ turistas } & \multicolumn{1}{c|}{ MRL } & \% error MRL & \multicolumn{1}{c|}{ SARIMA } & $\begin{array}{c}\text { \% error } \\
\text { SARIMA }\end{array}$ \\
\hline 2017:T1 & 2194190 & 1419230 & 35.3 & 2104310 & 4.1 \\
\hline 2017:T2 & 1150150 & 995754 & 13.4 & 1259700 & -9.5 \\
\hline $2017: T 3$ & 1413830 & 1061660 & 24.9 & 1478310 & -4.6 \\
\hline $2017: T 4$ & 1691720 & 1275540 & 24.6 & 1851770 & -9.5 \\
\hline $2018: T 1$ & 2150610 & 1474510 & 31.4 & 2367650 & -10.1 \\
\hline 2018:T2 & 1047190 & 1051040 & -0.4 & 1523040 & -45.4 \\
\hline 2018:T3 & 1133850 & 1116950 & 1.5 & 1741650 & -53.6 \\
\hline 2018:T4 & 1391280 & 1330820 & 4.3 & 2115110 & -52.0 \\
\hline
\end{tabular}

Fuente: Elaboración propia de los autores en base a datos de Subsecretaria de Turismo de Chile.

$\mathrm{Al}$ considerar las diferencias entre los pronósticos y los valores reales presentados en la tabla 3 respecto del turismo emisivo, se puede calcular un error promedio de 3,3\% en el caso del modelo MRL, y de un $-5.6 \%$ para el modelo SARIMA. Considerando los valores de la tabla 4 respecto del pronóstico del turismo receptivo, se puede calcular un error promedio de 16,9\% para el caso del modelo MRL, y de un $-22,6 \%$ para el modelo SARIMA. A primera vista se puede ver que el modelo MRL a pesar de no ser un modelo válido dado su comportamiento del residuo, entrega un error promedio menor que el obtenido por el modelo SARIMA; sin embargo, al observar la fig. 3 se puede apreciar que el modelo SARIMA entrega pronósticos muy cercanos a los valores reales para los primeros 5 trimestres en ambos casos, y luego diverge en forma importante en sus resultados, en particular, para el caso del turismo receptivo. Esta situación ha sido observada en este tipo de modelos que en general son útiles para estimaciones de corto plazo (Box et al., 2016:88). Realizando una estimación del error promedio cometido por el modelo SARIMA en un horizonte menor de tiempo, de sólo 5 trimestres, se obtiene un error promedio de $-5,9 \%$.

Los resultados muestran que no existen grandes diferencias entre el modelo MRL y el SARIMA para el caso del turismo receptivo; sin embargo, se debe recordar que el modelo lineal no es válido estadísticamente debido al comportamiento del residuo obtenido, que presenta una componente autorregresiva y no es de comportamiento normal N( $\mu, s)$. En cambio, en el turismo receptivo, la situación cambia en forma importante, y las estimaciones del modelo lineal son muy diferentes respecto de las obtenidas por el modelo SARIMA, en particular se puede ver que el MRL subestima en forma importante la tendencia de crecimiento de los valores futuros en el corto plazo y el efecto de la estacionalidad en el crecimiento del turismo receptivo. También se calcularon estadísticos de error para evaluar el grado de precisión de los modelos para sus valores pronosticados, los que se pueden observar en la tabla 5 .

Tabla 5: Estadísticos de evaluación de error del pronóstico.

\begin{tabular}{|c|c|c|c|c|}
\hline & \multicolumn{2}{|c|}{ Turismo emisivo } & \multicolumn{2}{c|}{ Turismo receptivo } \\
\hline Indicador & MRL & SARIMA & MRL & SARIMA \\
\hline EM & 32217 & -49902 & 305910 & -283590 \\
\hline RECM & 55788 & 70624 & 415740 & 389990 \\
\hline EAM & 38892 & 63696 & 306880 & 306060 \\
\hline PEM & 3.3 & -5.6 & 16.9 & -22.5 \\
\hline PEAM & 4.1 & 7.0 & 17.0 & 23.6 \\
\hline U de Theil & 0.37 & 0.41 & 0.70 & 1.10 \\
\hline
\end{tabular}

EM: error medio, RECM: raíz del error cuadrático medio, EAM: error absoluto medio, PEM: porcentaje de error medio, PEAM: porcentaje de error absoluto medio. Fuente: Elaboración propia de los autores en base a datos de Subsecretaria de Turismo de Chile. 
Los estadísticos de error muestran que los modelos MRL y SARIMA presentan rendimientos diferentes, y en general el modelo MRL tiende a subestimar los valores de turismo emisivo y receptivo, en cambio, el modelo SARIMA en general tiende a sobrestimar los valores de demanda turística. Además, los estadísticos de error muestran que, en general, ambas metodologías son más efectivas en modelar y pronosticar el turismo emisivo que el receptivo, en este último, el error aumenta para todos los indicadores considerados.

En resumen, los resultados muestran que los modelos SARIMA permiten pronosticar la demanda de turismo a pesar de presentar comportamientos no estacionarios y no lineal en su crecimiento y en su componente estacionalidad, y de mejor manera que los modelos MRL con variables dicotómicas estacional, entregando pronósticos bastantes fiables en el corto plazo. Estos resultados son similares a los obtenidos por Thushara et al. (2019) y por Ma et al. (2016) que mencionan que los modelos SARIMA proporcionan pronósticos precisos en presencia de estacionalidad y presentan errores menores al 10\% en promedio; y que en nuestro caso presentaron un error de -5,6\% para el turismo emisivo y de -5,9\% para el turismo receptivo, en un horizonte de corto plazo de 4 trimestres.

Estos resultados pueden ser útiles para el diseño de Políticas Públicas que requieran de pronósticos sobre el comportamiento de la demanda en el corto plazo y que pueden facilitar estimar las necesidades de ampliación de la capacidad de hospedaje o de transporte de turistas en determinadas temporadas; y con esto, determinar los recursos necesarios para enfrentar los posibles aumentos de la demanda turística del país.

\section{Conclusiones}

En este trabajo se ha modelado el turismo emisivo y receptivo que experimentó Chile para el período 2000-2016 utilizando datos trimestrales. Se han utilizado modelos de regresión lineal con variables dicotómicas para modelar la componente estacional presente en ambas series y los modelos ARIMAcon componente estacional propuestos por Box-Jenkins (1974). Los resultados muestran que los modelos SARIMA con componente estacional permiten modelar las series, que presentan un comportamiento no lineal en su crecimiento y con presencia de estacionalidad de mejor manera que los modelos de regresión lineal con componente estacional, entregando pronósticos bastantes fiables en el corto plazo, y con un error menor; -5,6\% para el turismo emisivo y de $-5,9 \%$ para el turismo receptivo en un horizonte de corto plazo. Estos resultados pueden ser útiles para el diseño de Políticas Públicas que requieran de pronósticos precisos sobre el comportamiento de la demanda turística, que faciliten la planificación del sector y, dimensionar en forma adecuada los recursos necesarios para enfrentar las fluctuaciones que puede experimentar la demanda turística en el corto plazo.

\section{Bibliografía}

Box, G. E., Jenkins, G., Reinsel, G. C., \& Ljung, G. M. 2016. Time series analysis: forecasting and control (5th ed.). New Jersey: John Wiley \& Sons.

Box, G. E. P., Jenkins, G. M., \& MacGregor, J. F. 1974. Some Recent Advances in Forecasting and Control. Applied Statistics, 23(2), 158. https://doi.org/10.2307/2346997

Chang, Y. W., \& Liao, M. Y. 2010. A seasonal ARIMA model of tourism forecasting: The case of Taiwan. Asia Pacific Journal of Tourism Research, 15(2), 215-221. https://doi.org/10.1080/10941661003630001

Chatziantoniou, I., Degiannakis, S., Eeckels, B., \& Filis, G. 2016. Forecasting tourist arrivals using origin country macroeconomics. Applied Economics, 48(27), 2571-2585. https://doi.org/10.1080/00 036846.2015.1125434

Choden, \& Unhapipat, S. 2018. ARIMA model to forecast international tourist visit in Bumthang, Bhutan. Journal of Physics: Conference Series, 1039(1). https://doi.org/10.1088/1742-6596/1039/1/012023

Du Preez, J., \& Witt, S. F. 2003. Univariate versus multivariate time series forecasting: an application to international tourism demand. International Journal of Forecasting, 19(3), 435-451. https://doi. org/10.1016/S0169-2070(02)00057-2

Goh, C., \& Law, R. 2002. Modeling and forecasting tourism demand for arrivals with stochastic nonstationary seasonality and intervention. Tourism Management, 23(5), 499-510. https://doi.org/10.1016/ S0261-5177(02)00009-2 
Goh, C., \& Law, R. 2011. The Methodological Progress of Tourism Demand Forecasting: A Review of Related Literature. Journal of Travel \& Tourism Marketing, 28(3), 296-317. https://doi.org/10.108 0/10548408.2011.562856

Greene, W. H. 2018. Econometric analysis (8th ed.). Pearson.

Higgins-Desbiolles, F. 2020. Socialising tourism for social and ecological justice after COVID-19. Tourism Geographies. https://doi.org/10.1080/14616688.2020.1757748

Kim, J. H., Wong, K., Athanasopoulos, G., \& Liu, S. 2011. Beyond point forecasting: Evaluation of alternative prediction intervals for tourist arrivals. International Journal of Forecasting, 27(3), 887-901. https://doi.org/10.1016/J.IJFORECAST.2010.02.014

Kulendran, N., \& Witt, S. F. 2003. Forecasting the Demand for International Business Tourism. Journal of Travel Research, 41(3), 265-271. https://doi.org/10.1177/0047287502239034

Kulendran, N., \& Wong, K. K. F. 2005. Modeling Seasonality in Tourism Forecasting. Journal of Travel Research, 44(2), 163-170. https://doi.org/10.1177/0047287505276605

Lim, C., \& McAleer, M. 2000. A seasonal analysis of Asian tourist arrivals to Australia. Applied Economics, 32(4), 499-509. https://doi.org/10.1080/000368400322660

Lim, C., \& McAleer, M. 2001. Forecasting tourist arrivals. Annals of Tourism Research, 28(4), 965-977. https://doi.org/10.1016/S0160-7383(01)00006-8

Lim, C., \& McAleer, M. 2002. Time series forecasts of international travel demand for Australia. Tourism Management, 23(4), 389-396. https://doi.org/10.1016/S0261-5177(01)00098-X

Ma, E., Liu, Y., Li, J., \& Chen, S. 2016. Anticipating Chinese tourists arrivals in Australia: A time series analysis. Tourism Management Perspectives, 17, 50-58. https://doi.org/10.1016/j.tmp.2015.12.004

Makridakis, S., \& Hibon, M. 1997. ARMA models and the Box-Jenkins methodology. Journal of Forecasting, 16(3), 147-163. https://doi.org/10.1002/(SICI)1099-131X(199705)16:3<147::AID- FOR652>3.0.CO;2-X

Organización Mundial del Turismo. 2017. Panorama OMT del turismo internacional, Edición 2017. In Panorama OMT del turismo internacional, Edición 2017. https://doi.org/10.18111/9789284419043

Peiris, H. 2016. A Seasonal ARIMA Model of Tourism Forecasting: The Case of Sri Lanka. Journal of Tourism, Hospitality and Sports, 22, 98-109. Retrieved from https://www.iiste.org/Journals/index. $\mathrm{php/JTHS/article/view/33831}$

Song, H., \& Li, G. 2008. Tourism demand modelling and forecasting-A review of recent research. Tourism Management, 29(2), 203-220. https://doi.org/10.1016/J.TOURMAN.2007.07.016

Song, H., Qiu, R. T. R., \& Park, J. 2019. A review of research on tourism demand forecasting. Annals of Tourism Research, 75, 338-362. https://doi.org/10.1016/J.ANNALS.2018.12.001

Song, H., Wen, L., \& Liu, C. 2019. Density tourism demand forecasting revisited. Annals of Tourism Research, 75, 379-392. https://doi.org/10.1016/J.ANNALS.2018.12.019

Song, H., \& Witt, S. F. 2011. Tourism demand modelling and forecasting: modern econometric approaches. Routledge.

Subsecretaria de Turismo de Chile. 2018. Estadísticas. Retrieved June 10, 2020, from http://www. subturismo.gob.cl/documentos/estadisticas/

Thushara, S. C., Su, J.-J., \& Bandara, J. S. 2019. Forecasting international tourist arrivals in formulating tourism strategies and planning: The case of Sri Lanka. Cogent Economics \& Finance, 7(1). https:// doi.org/10.1080/23322039.2019.1699884

Velásquez, J. D., Olaya, Y., \& Franco, C. J. 2010. Predicción de series temporales usando máquinas de vectores de soporte. Ingeniare, 18(1), 64-75. https://doi.org/10.4067/s0718-33052010000100008

Witt, C. A., Witt, S. F., \& Wilson, N. 1994. Forecasting international tourist flows. Annals of Tourism Research, 21(3), 612-628. https://doi.org/10.1016/0160-7383(94)90123-6

World travel \& Tourism Council. 2018. Economic Impact: Chile. Retrieved from https://www.wttc.org/ economic-impact/country-analysis/

Zenker, S., \& Kock, F. 2020. The coronavirus pandemic - A critical discussion of a tourism research agenda. Tourism Management, 81. https://doi.org/10.1016/j.tourman.2020.104164 


\section{ANEXO}

Tabla 6: Turismo emisivo y receptivo total de Chile (número de turistas, 2000-2018).

\begin{tabular}{|c|c|c|}
\hline Trimestres & Turismo emisivo & Turismo receptivo \\
\hline 2000:1 & 484801 & - \\
\hline 2000:2 & 273506 & - \\
\hline 2000:3 & 282440 & - \\
\hline 2000:4 & 278310 & - \\
\hline $2001: 1$ & 439487 & 704177 \\
\hline $2001: 2$ & 253590 & 310154 \\
\hline 2001:3 & 253954 & 310302 \\
\hline 2001:4 & 232960 & 435329 \\
\hline $2002: 1$ & 373100 & 497822 \\
\hline 2002:2 & 270531 & 265224 \\
\hline 2002:3 & 342219 & 301331 \\
\hline $2002: 4$ & 306915 & 426815 \\
\hline 2003:1 & 514566 & 510865 \\
\hline 2003:2 & 208578 & 300614 \\
\hline 2003:3 & 316355 & 358423 \\
\hline 2003:4 & 301291 & 502547 \\
\hline $2004: 1$ & 552374 & 596516 \\
\hline $2004: 2$ & 262002 & 342535 \\
\hline 2004:3 & 333431 & 377994 \\
\hline 2004:4 & 356469 & 507900 \\
\hline 2005:1 & 642086 & 677901 \\
\hline $2005: 2$ & 285057 & 351931 \\
\hline 2005:3 & 377379 & 442446 \\
\hline $2005: 4$ & 420864 & 597344 \\
\hline $2006: 1$ & 659952 & 754476 \\
\hline $2006: 2$ & 336351 & 426933 \\
\hline 2006:3 & 417874 & 450376 \\
\hline 2006:4 & 473005 & 642635 \\
\hline $2007: 1$ & 752348 & 827784 \\
\hline $2007: 2$ & 366776 & 469829 \\
\hline $2007: 3$ & 462100 & 526038 \\
\hline $2007: 4$ & 466130 & 710645 \\
\hline 2008:1 & 770357 & 939567 \\
\hline 2008:2 & 339143 & 462391 \\
\hline 2008:3 & 408262 & 562784 \\
\hline 2008:4 & 381760 & 745282 \\
\hline 2009:1 & 655541 & 973380 \\
\hline 2009:2 & 346791 & 513575 \\
\hline 2009:3 & 430059 & 507811 \\
\hline 2009:4 & 451600 & 764929 \\
\hline 2010:1 & 711899 & 921722 \\
\hline
\end{tabular}




\begin{tabular}{|c|c|c|}
\hline Trimestres & Turismo emisivo & Turismo receptivo \\
\hline $2010: 2$ & 406142 & 493969 \\
\hline 2010:3 & 501163 & 580275 \\
\hline 2010:4 & 599528 & 804671 \\
\hline 2011:1 & 829546 & 1031509 \\
\hline 2011:2 & 540020 & 593149 \\
\hline 2011:3 & 638717 & 638170 \\
\hline 2011:4 & 629312 & 874457 \\
\hline $2012: 1$ & 842192 & 1177875 \\
\hline 2012:2 & 598589 & 689356 \\
\hline 2012:3 & 700903 & 752624 \\
\hline 2012:4 & 695432 & 934424 \\
\hline $2013: 1$ & 905614 & 1191042 \\
\hline 2013:2 & 631339 & 660037 \\
\hline 2013:3 & 731781 & 751254 \\
\hline 2013:4 & 730011 & 973871 \\
\hline $2014: 1$ & 970312 & 1179078 \\
\hline $2014: 2$ & 674012 & 675609 \\
\hline $2014: 3$ & 761984 & 766401 \\
\hline 2014:4 & 762354 & 1053303 \\
\hline $2015: 1$ & 1068350 & 1363846 \\
\hline $2015: 2$ & 717753 & 861300 \\
\hline 2015:3 & 801952 & 926297 \\
\hline 2015:4 & 771029 & 1326893 \\
\hline $2016: 1$ & 1069169 & 1840963 \\
\hline 2016:2 & 748092 & 996352 \\
\hline 2016:3 & 885915 & 1214962 \\
\hline 2016:4 & 849685 & 1588423 \\
\hline $2017: 1$ & 1065603 & 2194193 \\
\hline $2017: 2$ & 763502 & 1150147 \\
\hline $2017: 3$ & 828179 & 1413828 \\
\hline 2017:4 & 962770 & 1691715 \\
\hline 2018:1 & 1108400 & 2150609 \\
\hline 2018:2 & 794784 & 1047190 \\
\hline 2018:3 & 968758 & 1133848 \\
\hline 2018:4 & 953124 & 1391281 \\
\hline
\end{tabular}

Recibido: 